\title{
Correction to: Expert Twisted: Event-Driven and Asynchronous Programming with Python
}

\author{
Mark Williams, Cory Benfield, Brian Warner, Moshe Zadka, \\ Dustin Mitchell, Kevin Samuel, Pierre Tardy
}

\section{Correction to:}

Chapter 7 in: M. Williams et al., Magic Wormhole, https://doi.org/10.1007/978-1-4842-3742-7_7

The original version of the book was inadvertently published without incorporating the author's proof corrections. The chapter has now been corrected and approved by the author.

The updated version of the chapter can be found at https://doi.org/10.1007/978-1-4842-3742-7_7 